\title{
Neuroscientific Basis of Decision Making Style among Potential Executives of Corporate World
}

\section{Santa Misra*}

Associate Professor and Head, Department Of Psychology, Utkal University, Odisha, India

"Corresponding author: Misra S, Associate Professor and Head, Department Of Psychology, Utkal University, Odisha, India, Tel: +91-9437211371; E-mail: santaamisraa@rediffmail.com

Received date: January 02, 2016; Accepted date: January 22, 2016; Published date: January 29, 2016

Copyright: (c) 2016 Misra. This is an open-access article distributed under the terms of the Creative Commons Attribution License, which permits unrestricted use, distribution, and reproduction in any medium, provided the original author and source are credited.

\begin{abstract}
Neuro-scientific research has now crowned the modern research era throughout the globe. Here is an attempt to focus on Cognitive Neuro science, of which Decision Making Style (DMS) is chosen as one of the imp aspect of it that affects our behavioral manifestation. Almost all activities, achievements, objectives, success and failure of our life is dependent on our decision making process. The multiple system of decision making lead to have a look to the reasons about why people differ in their decision making style. It leads to analyze Identification of core trade-offs imposed by a single-system solution to cognitive problems that are solved by multiple neural systems of Orbito Frontal Cortex, Anterior Cingulate Cortex, Amygdala, \& other complex neural-systems. With this idea the study aims at analyzing the implicit and explicit analysis of emotional and cognitive parts of Neuro-decision making among the potential executives in their summer training programme, of corporate engineer, to create awareness among the employees on the neuro scientific management of decision making, and its effects in the work place situations. Further out of several other components of DMS, like, Directive, intuitive, Behavioral, Spontaneous(Automatic), sensitive, Sequential, logical, Global, personable, Sensing, thinking, feeling , reasoning, emotional, etc., the Sequential, logical, Global, personable points were analyzed as the effective components of DMS. And an attempt has also made to build brain-based Neuro scientific models, capable of predicting decision making behaviors of the executives in organizational (HRM) sectors.
\end{abstract}

Keywords: Decision-making style; Multiple brain system; Neuro scientific models

\section{Introduction}

Neuro decision making Style of the employees in work environment has a great role in Human resource management of organizational sectors. Recent research on Neuro imaging technique with Neuro psychological and Neuro physiological basis of decision making style emphasizes on further investigations in preparing suggestive models that can help in controlling, modifying and managing the corporate world and to achieve their objectives.

Human beings, as the Homosapiens, are in the midst of a evolution. It has been 115 years since the neural cell was clearly recognized as the building block of the nervous system leading to the Neuro scientific interpretation of behavioral outburst. Blending of methods from neuroscience with management has seen surge of interest among the researchers. In recent trend of Neuro scientific researches. Impact of developments in neuroscience has inspired the trend of 'Brain Research' and its Interdisciplinary approach in combining social sciences and neuro science, that enables the analysis from physiology to neuroscience and from Neuro science to Neuro management. It links questions related to social, cognitive and brain mechanisms, via conventional neuroscience, neuroimaging and neuropsychological techniques.

Neuroscience, as an interdisciplinary science, involves the scientific study of theories and techniques of nervous system, work in living beings. Here focus is on Cognitive Neuro Science, of which Decision making style is chosen as one of the important component of our behavioral manifestation. Almost all activities, objectives, success and failure of our life is dependent on our decision making process. Hence Neuro Scientific Management of Decision Making (Figure 1) is to be focused for betterment of our life skill processing.

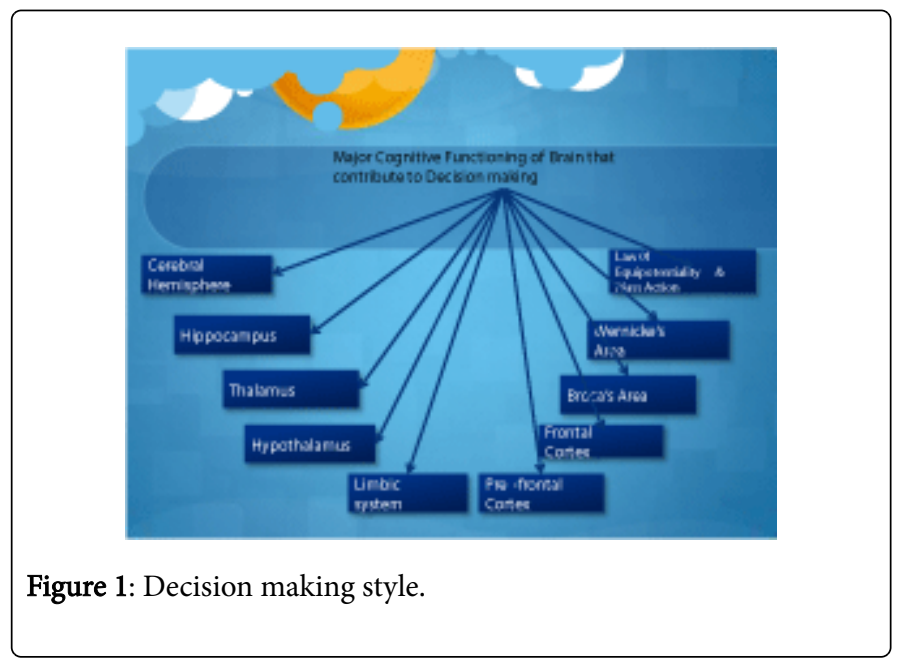

The Decision Making Style (DMS) is a cognitive process resulting in the selection of belief or course of action among several possible alternatives based on: (1) individuals way of thinking (some people tend to be rational, logical in the way they think \& some are cognitive \& intuitive); (2) individuals tolerance for ambiguity (some people have low tolerance \&some others have high tolerance for ambiguity) and (3) value based socio cognitive strategies. 
Every Decision-Making process produces a final choice that may or may not prompt action. Decision-Making is the study of identifying and choosing alternatives based on the values and preferences of the decision maker. Decision-making is one of the central activities of management and is a huge part of any process of implementation. The decision making can be considered from psychological, cognitive and normative point of view. The psychological aspect indicates context of a set of needs, preferences and values the individual has or seeks. When the Decision-Making process regarded as a continuous process integrated in the interaction with the environment is coming under cognitive component of decision making. The normative decision making involves the analysis of individual decisions concerned with the logic and rationality of decision-making and the invariant choice it leads to [1].

The fundamental questions that drive researchers in this area are how people make decisions; why people who are presented with same option make different choices; what is about cognitive \& neurological processes involved in DMS etc.

The answers can be expressed through various models of Decision Making. Human decision making models, which is having its origin from the time immemorial, is based on rationality, values, facts, means and ends that they observed.

In this context Reviews of Publications are analysed on the basis of:

Interactions between Cognition and Emotion in Decision Making

\section{Individual Differences in Decision Making}

\section{Social and Contextual Influences on Decision Making}

Since ancient times, scholars have studied Decision Making. But, in general, study of decision has been partitioned into three main approaches. For most economists, goal of studying decision behavior is prediction to develop formal mathematical models, typically based on a rigorous axiomatic foundation, which can predict decisions humans do, or should, make. These typically take as inputs state of external world and generate as outputs actual decisions made by human choosers. For an economist, a model is useful if it makes accurate predictions; whether or not the algorithm it employs mimics actual process of Decision Making is irrelevant to accomplishing this end. For this, managerial studies of Decision Making can be viewed as aimed towards achieving compact and abstract models of decision possible. The products are high-level, and often normative, theories that state testable Neuro hypotheses.

Over the course of the last three centuries, social and natural scientists have tried to understand how we make decisions, but using entirely different strategies. Since late 1990s, groups of interdisciplinary scholars have begun to combine social and natural scientific approaches to study of decision into an emerging synthetic discipline (Neuro-Managerial). The central assumption is that by combining both theoretical and empirical tools from neuroscience, psychology and managerial into a single approach, resulting synthesis will provide insights valuable to all parent disciplines. Studies conducted so far seem to support the conclusion. Theories from managerial and psychology have already begun to restructure neurobiological understanding of Decision Making. Recent findings are beginning to suggest constraints on theoretical models in psychological domains.

At a lower level of reduction, psychologists studying mechanisms of judgment and decision seek to understand mental constructs that guide decision making at a more process-based level of analysis. Mental processes like fear of losses or human tendency to overestimate low probabilities form algorithmic components of psychological models of decision. These models seek not just to predict behaviour but to capture accurately mental events that precede decision. Although this mental complexity often makes them more realistic it does so at a cost, because these models are so complicated they can often be hard to test completely. Neurobiologists have been trying to understand neural pathways and computations that give rise to decision-making behavior. These natural scientists have sought to understand, at a physical level, how it is that brain achieves decision by studying computational architecture of the brain.

During the past ten years, empirical studies of human choices in which uncertainty, inconsistency and incomplete information are present have produced a rich collection of findings which are beginning to be organized under broad generalizations. Since late $90 \mathrm{~s}$, interdisciplinary scholars have begun to combine social and natural scientific approaches to study of decision making into an emerging synthetic discipline called Neuro managerial. In 1998, less than 20 papers a year were published that included both 'brain' and 'Decision Making' as keywords. Since 2008, nearly 200 articles bearing those keywords have been published. In all probability, the first paper to openly combine neuro scientific data and mathematical theory was on 'Neural Computation of Utility' by Shizgal and Kent Conover's in 1996. The paper sought to describe neurobiological substrate for decision making using normative theory. In 1999 this was followed by Platt and Glimcher's publication of 'Neural correlates of decision variables in parietal cortex' which argued that: 'Neurobiologists have begun to focus increasingly on study of sensory-motor processing, but many describe these processes remain rooted in classic reflex' and went on to 'describe a formal managerial-mathematical approach for physiological study of sensory-motor process, or decision making. Within neurobiological circles this paper was rapidly followed by a suite of papers uniting both managerial and psychological theories of decision making with measurements in human brains.

The first formal paper in neuro managerial was published by [2]. The paper employed psychological Prospect theory of decision making developed by Kahneman and Tverskyin and brain scanning experiment. The scanning experiment revealed that brain activation in ventral striatum matched predicted subjective valuations. The second reflected collaboration between McCabe and Smith. This represented use of game theory in human neurobiological experiment data. Critical insight was that decision-making systems of brain can be viewed as fundamentally two-part system. Areas in frontal cortex and basal ganglia form first of these two parts. These areas learn and compute values of available actions and are a set of valuation structures that these areas principally contribute to decision-making. Outputs of these then appear to pass to fronto-parietal circuits that actually 'decide' between options based on these antecedent valuations and pass these decisions on to motor system for implementation. Subsequent studies have largely supported segregation of neural architecture into valuation and decision making systems, although levels of interconnection between these two are being explored.

Glimcher [3] directed that reviewed history of neuroscience and argued that history was striking in its lack of normative models for higher cognitive function [4,5]. In essence, they argued that insights into biological mechanism are unlikely to have much impact on its psycho-social manifestations. 
Glimcher [3] argued that the mathematically specified mechanism does not exist thus only weakly contravenes the theory. While there is one candidate demonstration of this type emerging in the literature authored by [6], the demonstration that neurobiological data can shape managerial theories of behaviour remains incomplete.

In addition to these research centers, 'The Society for Neuro managerial serves as a central focus for the emerging discipline. In 2009, the Society published, in collaboration with Academic Press, 'Neuromanagerial Decision Making and the Brain'. It summarizes current advances and controversies in the field and serves as a starting point for anyone interested in learning more about this academic discipline.

Peter [7] reviewed in 'Current Directions in Psychological Science: on the neural computation of Utility. The publication sought to describe neurobiological substrate for decision using normative managerial model [8] publication of 'Neural correlates of decision variables in parietal cortex, which argued that: 'Neurobiologists have begun to focus increasingly on study of sensory-motor processing, but models used to describe these processes remain rooted in classic reflex' and went on to 'describe a formal managerial-mathematical approach for physiological study of sensory-motor process, or decision-making'. Empirically, the publication demonstrated that activity of individual neurons in posterior parietal cortex encoded both probability and magnitude of reward as would be predicted by most managerial theories if these neurons participated in decision-making. Within neurobiological circles this publication, which sought to use managerial approaches to studying decision, was rapidly followed by a suite of publications in managerial and psychological theories of decision with measurements in human brains.

\section{Rationale}

Although there is a general agreement that people make decision by using assumptions, beliefs, habits, based on mentality, ethics, values and attitudes, neuro psychologists suggest that brain considers various sources of information in making a decision and it is the neuro physiological content that determine the permutation and combination of decision making style of a person. Hence the present paper (a part of post doctoral research work on exploration of Neurodecision-making style) explores the physiological aspect of DMS \& its role within a new framework of investigation called neuro-scientific management in the process of decision making style and accordingly to prepare a model that can help the people in managing their decision making process in differential environmental conditions. Since rational models like classical DM model and cognitive naturalistic modal do not always predict accurately an individual's behaviour, emphasis has been given to several other models for the purpose.

\section{Objectives}

Thus the study is an attempt -

To build brain-based models, capable of predicting decision making behavior and to provide a conceptual framework for understanding and conducting research on Neuro - management perspectives in decision making styles of potential executives in corporate world.

To analyze different components of the decision making style of potential executives in their work place relating to Human Resource Management (HRM)
And based on these advocacies to prepare an exemplary model with inviting the suggestions from various research stand points.

\section{Methodology}

\section{Sample}

100 Ss from Tier 1 corporate in India, joined as Assistant System Engineer (ASE), in their training period at - NalSarovar - ILP Center, 2nd, 3rd \& 4th Floor, Info Tower - III, Infocity, Gandhinagar - 382 009, Gujarat, India, who are considered as potential executives with their average income range - 3-4 lakhs per annum.

\section{Instruments used}

The Decision making style questionnaire by "Co-operative Education and career Division" was used for data collection which consists of 24 questions in a five point scale strongly disagrees, disagree, neutral, agree, and strongly agree. The components of decision making analyzed were Rational, dependent, avoidant, intuitive and spontaneous.

My Decision making style questionnaire consisting of six questions with four alternative choices, the domains analyzed were sequential, logical, global and personable.

\section{Procedure}

This study is an extension of the previous study on Decision making style of potential executives of TCS group, where only Rationale, Dependent, Avoidant, Intuitive and Spontaneous components of decision making style were analyzed But in the present study some more components are added to strengthened the result on individual decision making style, like, sequential, logical, global and personable.

All the subjects were individually administered the Decision making style questionnaires in their own set up. After the data collection the result was analysed, based on these nine components of decision making process.

\section{Analysis of result}

The result was interpreted on rational decision making, intuitive decision making, dependent decision making, avoidant decision making, and spontaneous decision making.

Rationale: This approach is characterized by using a logical and structured approach to decision making. One may find using ideas such as SWOT or force field analysis helpful here, which are explained later in this section. The average score of all the subjects in this component is $30 \%$.

Dependent: This approach is characterized by reliance upon the advice, direction and support of others. You will find that you are more comfortable making a decision when you have discussed the options with others, and are uncomfortable making decisions alone. The average score of all the subjects in this component is $18 \%$.

Avoidant: This approach is where one attempt to postpone or avoid making a decision. This is not a healthy way to approach making decisions. Whilst taking time to reflect on the options is a good idea, avoiding or postponing making the decision can lead to negative consequences. The average score of all the subjects in this component is $14 \%$. 
Page 4 of 5

Intuitive: This approach is characterized by reliance upon hunches, feelings and impressions. One will go with 'gut instinct' or with what feels right, rather than taking a logical approach to the decision making/ The average score of all the subjects in this component is 18 $\%$.

Spontaneous: This is where the decision maker is impulsive and prone to making 'snap' or 'spur of the moment' decisions. This can be a valuable trait in terms of not over planning the future, but it is not always a good idea to leave important decisions to be made this way. The average score of all the subjects in this component is $20 \%$ (Table $1)$.

\begin{tabular}{|l|l|l|l|l|l|}
\hline Components of DSM & Rational & Dependent & Avoidant & Intuitive & Spontaneous \\
\hline Percentage of efficiency & $30 \%$ & $18 \%$ & $14 \%$ & $18 \%$ & $20 \%$ \\
\hline Percentage of deficiency & $70 \%$ & $82 \%$ & $86 \%$ & $82 \%$ & $80 \%$ \\
\hline
\end{tabular}

Table 1: Showing \% of efficiency and deficiency in different components of decision making style of 100 potential executives.

The percentage of scores in Rational Decision Making, intuitive decision making dependent decision making, avoidant decision making, and spontaneous decision making were found to be $30 \%, 18 \%$, $14 \%, 18 \%$, and $20 \%$, respectively. Less dependent component (18\%) can be considered as better quality of an administrative officer. But their deficiency level in these components, mainly on rational, avoidant, intuitive, and spontaneous, which are important characteristics for administrative personnel, are found to be $70 \%(86 \%$, $82 \%$ and $80 \%$ ). Hence the causal effects of these points can be taken into consideration and can also be explained on the basis of neurophysiological basis of their behaviour.

In analyzing the second questionnaire, that can help in supporting previous finding of the author, the components used are: sequential, logical, global and personable.

Sequential: Sequential decision makers need lots of specific information, the details of what's being asked, instructions on the best way of doing things, evidence that particular procedures work best, and steps for doing the task correctly.

Logical: Logical decision makers want the specifics, but more than that they want reasons, defensible positions, and a clear understanding of the possible results of the different choices. They tend to balance off one set of choices in relationship to the others. They exercise objective and critical judgment in order to not make choices based on personal feelings.

Global: Global decision makers want to explore all the possibilities. This process includes what exists as well as that which can be imagined. They need the specifics, but more than that they need a feeling of "fit", of elegance, of an expanded and more inclusive view of what's possible.
Personable: Personable decision makers need lots of specific information, good problem definitions, and the sharing of other people's experiences. But even more than that they need to explore their own and other people's feelings about the decision(s) being faced. They look for ways to draw out their own and others' values. They want the decision-making process to be collegial, cooperative, and sensitive to the individual's needs. They decide best where the environment is relaxed, friendly, and supportive of individual needs. They need to talk extensively to make sure each person's point-of-view has been expressed and heard. They need continual verbalizations of steps taken and conclusions drawn. They search for both consensus and a feeling of "group ownership" of process and conclusion.

The percentage of scores in these four components are $27 \%, 29 \%$, $24 \%$, \& $21 \%$ respectively. This cross check on the components of decision making style in both the questionnaires of all 100 potential executives revealed the idea that the subjects who are having more score in rational (30\%) components also kept highest score (29\%) in Logical component .Those having next highest score in spontaneous (20\%) also kept the next highest in sequential component (27\%) which are almost similar by nature. The rest two components global (24\%) and personable (21\%) are two additional assessments of DMS components which refer the efficiency level of the subjects in work place situations. If all the possibilities will not be explored by the executives they can't make accurate steps in their administrative pursuits (Global) and if they will not have lots of specific information, good problem definitions, and the sharing of other people's experiences then it can be a question to the company and management (Personable) (Table 2). Hence subjects proved to be the fit-in potentialities in executive posts for the corporate world (Figure 2).

\begin{tabular}{|l|l|l|l|l|}
\hline Components of DSM & Sequential & Logical & Global & Personable \\
\hline Percentage of efficiency & $27 \%$ & $29 \%$ & $24 \%$ & $21 \%$ \\
\hline Percentage of deficiency & $73 \%$ & $71 \%$ & $76 \%$ & $79 \%$ \\
\hline
\end{tabular}

Table 2: Showing \% of efficiency and deficiency in different components of Decision Making Style of 100 potential executives. 
Page 5 of 5

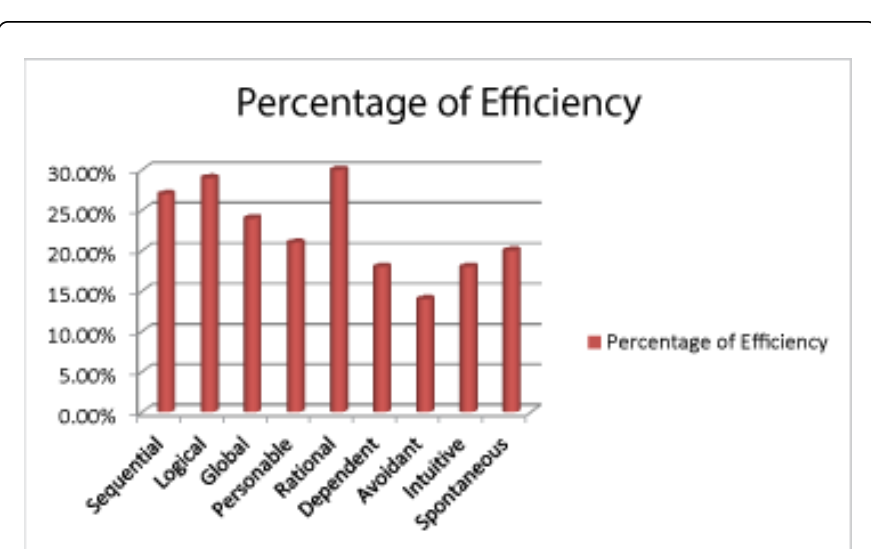

Figure 2: Showing the percentage of efficiency in all nine components of DMS.

\section{Conclusion}

There is Neuro- Scientific acceptance to decision making style about the involvement of the brain to take a decision as per the review of literature

The nine components of decision making style discussed are inevitable part of taking a decision

The executive personnel with better scores of the discussed components can be the assets for the corporate world.

Based on such types of explorations in this era, brain based models can be formed that can act as an exemplary in developing, improving and managing the decision making capacity of the employees in different job sectors (Figure 3).

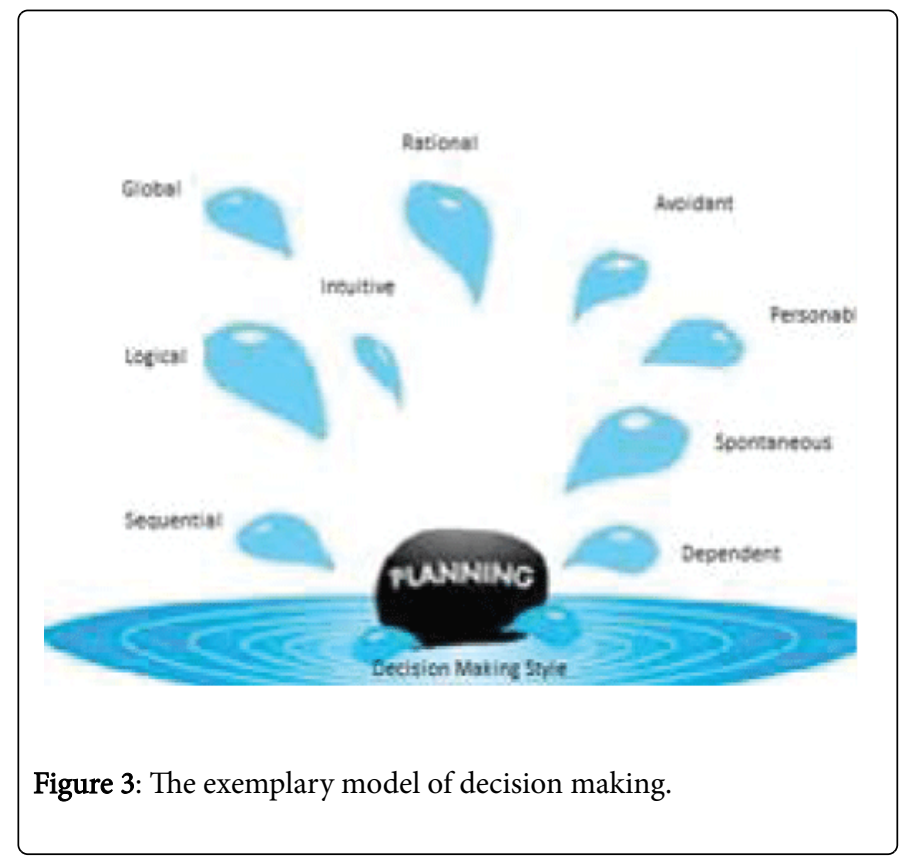

\section{The likely contributions of the study are}

Provides a conceptual and philosophical framework for understanding and conducting Neuro-managerial research at the intersection of neuroscience, managerial \& psychology.

Describes a standard model for decision process that links and spans neurobiological, psychological and managerial levels of analysis.

Applies neuroscience to Neuro-managerial and ties both fields to biological constraints in how we judge relative value and make decisions.

An important resource for researchers in interdisciplinary research.

\section{References}

1. Kahneman D, Tversky A (1979) Prospect Model: An Analysis Of Decisions Under Risk. Econometrica 47: 262-291.

2. Breiter HC, Etcoff NL, Whalen PJ, Strauss MM, Rauch SL, et al. (1996) Response and habituation of the human amygdala during visual processing of facial expression. Neuron 17: 875-887.

3. Glimcher PW (2003) Decisions, Uncertainty, And The Brain: The Science Of Neuro - Management Decision Making, MIT Press.

4. Faruk G, Wolfgang P (2008) Measurable ambiguity. Levine's working paper Archives .

5. Loewenstein C, Colin G, Drazen P (2005) Neuroeconomics: How Neuroscience Can Inform Economics. Journal of Economic Literature 43: 9-64.

6. Mauricio RD, Schotter A, Ozbay EY, Phelps EA (2008) Understanding overbidding: Using the Neural Circuitry of Reward to Design Economic Auctions. Science 321: 1849-1852.

7. Peter S, Kent C (1996) On the neural computation of utility. Psychological Science 5: 37-43.

8. Platt ML, Glimcher PW (1999) Neural Correlates Of Decision Variables In Parietal Cortex. Nature 400: 233-238. 